\title{
La teoría de sistemas de Niklas Luhmann
}

\author{
Niklas Luhmann's systems theory \\ EGUZKI URTEAGA \\ Universidad del País Vasco \\ Departamento de Sociología 1
}

Recibido: 26-11-2008 Aprobado definitivamente: 22-01-2009

\section{RESUMEN}

Sociólogo alemán profundamente original y difícilmente clasificable, Niklas Luhmann (19271998) ha elaborado una teoría ambiciosa y coherente en la que describe la sociedad moderna como un sistema. Constituido, no tanto por individuos sino por comunicación, se diferencia en subsistemas funcionales cerrados a través de códigos especializados: los sistemas político, económico, religioso, artístico o jurídico. Inspirándose en autores, teorías y disciplinas muy diferentes, Niklas Luhmman ha construido una de las obras más fecundas y singulares del siglo $\mathrm{XX}$. De manera más precisa, el objetivo de este artículo es analizar su teoría de los sistemas.

\section{PALABRAS CLAVE}

LUHMANN, TEORÍA, SISTEMA, SUBSISTEMA, DIFERENCIACIÓN

\begin{abstract}
The original and hardly classifiable German sociologist Niklas Luhmann (1927-1998) elaborated an ambitious and coherent theory which describes modern society as a system. Constituted, not so much of individuals but of communication, it is divided in closed functional subsystems through specializing codes: the political, economic, religious, artistic or juridical systems. Inspired by authors, theories and very different disciplines, Niklas Luhmman constructed one of the most fecund and singular works of the XX century. More precisely, the target of this article is to analyze his systems theory.
\end{abstract}

\section{KEYWORDS}

LUHMANN, THEORY, SYSTEM, SUBSYSTEM, DIFFERENTIATION 


\section{INTRODUCCIÓN}

La obRa DE Niklas LuHMann fascina a los sociólogos por su ambición teórica poco común. A pesar de la diversidad de objetos de estudio, desarrolla una visión global de la sociedad, que se extiende de la pedagogía a la globalización, de los medios de comunicación a la moral, de la ecología a la semántica. Esta diversidad se nutre de numerosas lecturas. Utiliza un material diverso que consta tanto de la literatura francesa del siglo XVII como de la prensa diaria brasileña o de ensayos de ciencias cognitivas. Ello explica que su propósito trascienda las fronteras disciplinares.

La teoría del sociólogo alemán es compleja y la dificultad no se limita al estilo de su obra. Su finalidad no consiste únicamente en la transformación de los hechos en problemas y del mundo en improbabilidad, sino que además el estilo mismo de los libros de Luhmann se caracteriza por su aridez. Se suceden largos pasajes de abstracción teórica que parecen estar completamente desconectados de la realidad empírica, pero, de pronto, se interpone, en una frase, una nota o una ilustración, una escena trivial. Asimismo, el modo de exposición de Luhmann no es lineal. Los capítulos de sus ensayos, especialmente los últimos, pueden leerse en cualquier orden y Luhmann procede por toques, acumulaciones y yuxtaposiciones. Así, la opinión pública ha sido el objeto de tres publicaciones sucesivas, que se extienden desde 1971 hasta 2000.

La trayectoria de Niklas Luhmann es insólita en el mundo académico alemán. Tras la Segunda Guerra Mundial, estudia Derecho en la Universidad de Friburgo, antes de convertirse en funcionario del Ministerio de Educación y Cultura del Land de Baja-Sajonia. Después de diez años en la Administración, coge un año sabático para estudiar en la Universidad de Harvard. Allí se encuentra con Talcott Parsons y descubre el funcionalismo, el cual le ofrece sus primeras categorías analíticas. Se relaciona igualmente con Jürgen Habermas con el cual mantendrá un polémico diálogo una década más tarde. No obstante, esta relación se prolongará hasta la muerte de Luhmann (1998). A su regreso a Alemania en 1962, consigue un doctorado y una habilitación, bajo la dirección de Helmut Schelsky. Después de haber impartido docencia en la Universidad de Munster, es nombrado profesor en la Universidad de Bielefeld, donde permanecerá hasta el final de su carrera académica.

Sus trabajos iniciales se centran en la ciencia de la administración y el derecho. En la década de 1970, empieza a explorar las esferas sociales una tras otra: la religión, la política, la ciencia, la economía, antes de fijar la estructura teórica y epistemológica de su reflexión en Soziale Systeme en 1984 y de nuevo en 1997 en Die Gesellschaft der Gesellschaft. En un segundo periodo, teoriza de manera metódica la sociedad como sistema, un sistema constituido únicamente por la comunicación. El sistema social reproduce la comunicación tal y como 
los sistemas vivos reproducen la vida y los sistemas psíquicos reproducen la conciencia. Todo lo que no es comunicación pertenece a su entorno. Como cualquier sistema está cerrado sobre sí mismo, el individuo carece de medios para intervenir sobre el sistema social y, más aún, para gobernarlo. Paralelamente, el proceso de diferenciación que ha acompañado a la modernidad es concebido a través de la constitución de subsistemas opacos que se perciben mutuamente como elementos del entorno.

Este razonamiento responde a una preocupación principal: la complejidad, cuyo control se sitúa en el fundamento de la lógica evolutiva de los sistemas. En la década de 1990, esta problemática, sin desaparecer completamente, es sustituida progresivamente por las ideas de observación y de distinción, que le permiten reformular la teoría de los sistemas como una teoría que contempla los demás sistemas como observadores. No solamente Luhmann no vuelve sobre lo que ha escrito, reeditando sin revisar y publicando artículos de diferentes periodos, sino que los desplazamientos se producen, como el del binomio sistema/entorno hacia el de forma/medio.

Luhmann rompe con el presupuesto de que hay un actor o una acción detrás de la comunicación social. Va más allá, al no considerar cualquier proyecto teórico como una identidad (el sistema) sino como una diferencia (entre el sistema y su entorno). El sistema no existe en sí mismo sino que sólo existe y se mantiene gracias a su distinción con el entorno. Pero, el valor de la diferencia es relativo al sistema considerado. Dicho de otra forma, es cuestión de abandonar el viejo sueño de comprender el mundo como una unidad, desde una posición casi divina, y de descubrir un sentido unificado tras la sociedad, haciendo derivar la sociedad de la naturaleza del hombre o de un contrato, para concentrarse en una comprensión del mundo en términos de redes de observadores cruzados y horizontales, que no pueden ser unificados por una observación totalizada.

La teoría luhmanniana de los sistemas intenta ofrecer un instrumento rigurosamente coherente de descripción de los sistemas, sean orgánicos o inorgánicos, y de pensar su aplicación en ciertos ámbitos. A pesar de ello, su obra es objeto de cierto silencio tanto en Francia como en España, mientras que ha suscitado numerosos discusiones en Alemania, Italia, los países nórdicos y, un poco más tarde, en Estados Unidos. Si las disciplinas jurídicas tanto en Francia como en España se han interesado por algunas de sus obras y sus principales libros han sido objeto de una traducción progresiva, esta tímida recepción no tiene comparación alguna con la importancia de la que goza en Alemania. En España, su «hostilidad ante la ilusión ética» o su observación sin pathos del mundo aspirado por la lógica del control de la complejidad no han desatado ninguna polémica. En otros países, sin embargo, le reprochan su tendencia a describir la sociedad sin criticarla y sin pretender corregir los problemas sociales y ecológicos generados por su evolución. 
No obstante, los textos de Luhmann no están desprovistos de enunciados prescriptivos o normativos. La ubicación del individuo en el entorno del sistema no se produce a partir de una neutralidad científica, sino que se articula entorno a la idea de la orientación de la sociedad a partir de una concepción normativa del ser humano, que caracteriza la modernidad. Ello está en el origen de las experiencias negativas que caracterizan este periodo, como el endurecimiento de las asimetrías de los roles en distinciones entre electos y damnificados. La teoría de Luhmann proclama el agotamiento de las categorías heredadas de la modernidad, en particular sus categorías normativas, y ofrece un diagnóstico pesimista de una sociedad que no dispone de ninguna posibilidad de modificar su trayectoria, encaminándola hacia el desastre ecológico.

Más precisamente, este artículo pretende analizar un aspecto de la obra de Niklas Luhmann: su teoría de los sistemas, que se caracteriza por su carácter transdisciplinar, su distinción de tres sistemas, su percepción del sistema social como un sistema diferenciado en las sociedades modernas y el carácter auto-referencial o autopoiético de los mismos. Efectivamente, el mundo está constituido únicamente por unos sistemas que perciben los acontecimientos que se producen en sus entornos como ruidos. Utiliza y distingue tres grandes tipos de sistemas: el sistema vivo, el sistema psíquico y el sistema social. El primero se reproduce gracias a la vida, el segundo lo hace vía la conciencia y el tercero se perpetúa a través de la comunicación. El interés de Luhmann se centra en este último, en la medida en que las sociedades modernas se caracterizan por una diferenciación de sus sistemas en subsistemas, entre los cuales figuran los subsistemas político, económico, artístico, religioso o educativo. Cada uno es autopoiético y no se trata de una estructura o un conjunto ordenado de elementos determinados. El sistema está cerrado por sus propias operaciones y su entorno solo le afecta en la medida en que lo ha determinado.

\section{UN MODELO TRANSDISCIPLINAR}

La investigación celular y neurobiológica es esencial para el desarrollo de una teoría de los sistemas operativos cerrados, puesto que es sobre la base del modelo de un sistema nervioso incapaz de incidir sobre su propio entorno, es decir que no controla la causalidad que sus propias operaciones generan en el entorno, sobre la que Luhmann construye su concepción de los sistemas. Concierne tanto al sistema psíquico, incapaz de alcanzar otro sistema, como al sistema social, incapaz de controlar los efectos que genera en su entorno natural, como a la interrelación de los subsistemas sociales entre si. Efectivamente, la investigación sobre el cerebro ha demostrado que puede estar acorde con su entorno sin apenas entrar en contacto con él. Ello implica que «el conocimiento no es una representación del entorno en el sistema, sino que, al contrario, es la 
producción de construcciones propias, que no está estructurada por el entorno pero que puede simularlo». ${ }^{1}$

El sistema no mantiene ningún contacto con su entorno al nivel de sus operaciones y, en el seno del sistema, nada se parece a unos signos que constituirían referencias para los elementos situados en su exterior. No obstante, este cierre puede ser simulado dentro del sistema. Luhmann califica esta simulación de reentry. El concepto de autopoiesis, que se halla en el centro de la sociología sistémica, resulta de los trabajos de Maturana y Varela. ${ }^{2}$ Estos han intentado explicar la manera según la cual un sistema se auto-reproduce, y el término de autopoiesis designa la realización de la perpetua auto-afirmación factual del sistema ante su entorno. Maturana ha elegido el término griego de poiesis, en su acepción de actividad concreta, en oposición a la praxis dotado del sentido de una acción adosada a una reflexión, con el fin de significar claramente que el sistema es la obra de su propio trabajo. Este concepto de autopoiesis se asocia a la noción de «cerradura operativa». No se trata ni de una propiedad ni de un conjunto de elementos que cierra el sistema y traza sus límites, sino de una cadena de operaciones. Los elementos están pensados como efectos del sistema.

Estas premisas han sido posibles gracias a una larga construcción teórica iniciada después de la Segunda Guerra mundial que indica un cambio de paradigma científico. Después de haber intentado circunscribir la complejidad, a través de unos análisis estadísticos y de la teoría de la probabilidad, intentan dar cuenta de una complejidad ineluctable. La teoría de los sistemas representa un intento por abordar este problema desde una óptica transdisciplinal. Se trata de elaborar una teoría y una metodología única. El origen de la teoría de los sistemas se encuentra en los inicios de la cibernética. Se discuten los mecanismos de feed-back y de causalidad circular en los sistemas biológicos y sociales. Lo que está en juego es la teorización de estas retroacciones negativas en el seno de las cuales A afecta a B en el mismo momento en el cual se ve afectado por sus efectos sobre B. Siguiendo este camino, la teoría de los sistemas cuestiona los conceptos científicos tradicionales de causalidad, de determinismo y de reduccionismo, sustituyéndolos por las nociones de causalidad circular, de auto-organización y de indeterminación, antes de orientarse progresivamente hacia la demostración y la elucidación de la emergencia imprevisible del orden a partir del desorden.

Efectivamente, si inicialmente la teoría de los sistemas pone el énfasis en la estabilidad y en la capacidad de la circularidad por garantizarla, la segunda generación de teóricos se interesa por la manera según la cual la recursividad puede generar efectos sistémicos no esperados. Von Foerster, por su parte,

1 Duprat, G. 1990: Connaissance du politique. Paris: PUF, p. 285.

2 Maturana, H., Varela, F. 1980: Autopoïesis and Cognition. Boston: D. Reidel. 
reflexiona sobre la relatividad de la observación. De hecho, añade a este modelo la idea que el mundo no está dado sino que emerge de la interacción del observador y del observado. Cualquier descripción implica al que describe. En otros términos, desarrolla la idea que cualquier observación esconde su origen y consta, por lo tanto, de una «mancha ciega» que solo puede desaparecer con otra observación, es decir con la creación de otra «mancha ciega». El enfoque teórico general de Luhmann se deja percibir como un intento para definir unos conceptos interrelacionados.

\section{LA ARQUITECTURA LUHMANIANNA}

Luhmann distingue tres tipos de sistemas comparables y comparados: el sistema vivo, el sistema psíquico y el sistema social. Estos sistemas consisten únicamente en acontecimientos: eventos de pensamiento para el sistema psíquico, eventos de comunicación para el sistema social y eventos de suspensión de la muerte para el sistema vivo. Se producen acontecimientos en un momento determinado y desaparecen tan rápidamente como han aparecido, haciendo un llamamiento a otros que suceden para que el sistema pueda perpetuarse. Nos centraremos exclusivamente en el sistema social.

En la teoría sistémica luhmanniana, la comunicación produce y reproduce la sociedad. La comunicación constituye una operación sistemática e ineluctablemente de carácter social que implica, al menos, dos socios cuyas acciones solo pueden ser constituidas por la comunicación. La expresión sistema social hace referencia, en este caso, a la sociedad en su conjunto en la medida en que reproduce la comunicación por la comunicación. Según Luhmann, la sociología siempre ha concedido demasiada importancia a las diferencias culturales, nacionales y regionales. Solo es cuestión de diferenciaciones internas, que son secundarias a la hora de comprender la manera según la cual la sociedad actúa, se transforma y produce acontecimientos. El principio de diferenciación que estructura verdaderamente la sociedad moderna es funcional.

La idea de sistema social se aplica igualmente, aunque sea de manera marginal, a otras entidades de la sociedad: a las organizaciones, por una parte, y a sus interacciones, por otra. Las organizaciones son sistemas sociales autopoiéticos, en el seno de los cuales las únicas operaciones posibles son las decisiones. A pesar de que éstas son secuencias de acontecimientos que permiten la siguiente decisión, no puede hablarse de una finalidad de la organización a la que estarían subordinadas. Como sistema autopiético, una organización tiene fronteras y elementos que no son datos objetivos sino productos propios de la organización. Asimismo, cualquier interacción constituye un sistema social, cerrado operativamente. Estos dos tipos de sistemas, la interacción y la organización, forman parte de la sociedad pero su suma no equivale a un sistema social global. 
La comunicación que la constituye no es necesariamente verbal y no se resume a un simple anuncio, ni puede comprenderse como la pura transferencia de informaciones. El procedimiento de comunicación es, para Luhmann, la síntesis de una información, de un enunciado y de una comprensión. La información se define como una «distinción que diferencia un acontecimiento posterior». Dicho de otra forma, es una distinción que cobra sentido y puede ser conectada a otra. La comunicación solo se produce cuando alguien mira, escucha, lee y comprende lo suficiente como para que la comunicación pueda producirse ${ }^{3}$. En cuanto al anuncio, se distingue de la información por su manera de responder a la necesidad y de no hacer depender la continuidad de la comunicación, de la pertinencia y de la calidad de la información. Esto no significa que cualquier comunicación interna al sistema sea necesariamente exitosa, ya que la incomprensión y el rechazo de comunicar no constituyen una salida del sistema sino que forman parte del proceso.

No obstante, el sistema social, que se extiende al conjunto de las comunicaciones de la sociedad mundial, no está exento de diferenciaciones.

\section{LA DIFERENCIACIÓN DEL SISTEMA SOCIAL}

El sistema social se divide en subsistemas: el sistema político, el sistema económico, el sistema científico, el sistema religioso, el sistema artístico, el sistema mediático, el sistema educativo y el sistema familiar al que añade posteriormente el sistema jurídico. Efectivamente, durante un largo periodo, el derecho solo es concebido como una estructura del sistema político y solamente aparece como un sistema específico a partir de la década de años 1980. La aparición de los subsistemas se corresponde con la necesidad de la sociedad de alcanzar un nivel superior de complejidad: «se puede describir una sociedad como funcionalmente diferenciada a partir del momento en el cual forma sus principales subsistemas en la perspectiva de problemas específicos que deberán ser resueltos en el marco de cada sistema funcional». ${ }^{4}$ Es la razón por la cual, para Luhmann, la diferenciación funcional es el principal criterio para distinguir las sociedades modernas de las sociedades que las preceden.

En el seno de las sociedades premodernas, prevalecen unas formas de diferenciación poco complejas. Luhmann distingue tres tipos que han podido existir tanto sucesivamente como de manera concomitante en el seno de una misma sociedad. La diferenciación basada en la descendencia o la residencia, se caracteriza por el hecho de que la comunicación se limita a las relaciones de cara a cara. La diferenciación centro-periferia implica una diferenciación

3 Bateson, G. 1972: Steps to an Ecology of Mind: A Revolutionary Approach to Man's Understanding of Himself. New York: Ballantine, p. 272.

4 Luhmann, N. 1999: Politique et complexité. Paris: Cerf, p. 43. 
desigualitaria de tipo civilizado/no civilizado. Hace posible una difusión territorial de la comunicación. Por último, la diferenciación por estratos, que está vinculada a la existencia de una aristocracia, permite un mayor nivel de complejidad, en la medida en que la casta superior asume la responsabilidad de realizar la auto-descripción de la sociedad.

Las sociedades organizadas en función de estas formas de diferenciación premodernas tienen en común el hecho de estar estructuradas jerárquicamente, ya que están representadas por una parte de ellas-mismas. Al contrario, la sociedad moderna funcionalmente diferenciada se caracteriza por la ausencia de jerarquía y de control del centro. Esto no significa que, en la sociedad moderna, no existan órdenes de subordinación o de desigualdades sino que estos no resultan de la estructura primaria de la sociedad: una función deja de depender estructuralmente de su relación con las demás funciones.

La diferenciación en subsistemas funcionales se comprende a través del modelo de la producción por el sistema de lo que lo constituye y de lo que lo limita. Puesto que el sistema social está formado por la comunicación, la evolución hacia la diferenciación depende de la producción de semánticas autónomas, y se traduce por la aparición de secuencias de comunicaciones propias a cada subsistema. Además, cada subsistema observa la sociedad a partir de su propia función. Esta observación, según la cual se orienta, está estructurada por una distinción binaria, que no admite interferencias en la realización de la función: el código. Así, el sistema científico tiene como código la distinción verdad/ falso, mientras que el sistema jurídico hace la distinción entre lo legal y lo ilegal. Cualquier información de un sistema es tratado a través de este código. Cada sistema funcional selecciona en función de sus propias distinciones. Por ejemplo, el sistema económico no deja lugar a ninguna orientación por parte de la ciencia.

Distinguiendo los principales subsistemas según sus funciones respectivas, se puede analizar la lógica que los mantiene unidos y separados.

1. La ciencia está especializada en los avances cognitivos y en los procesos sociales de aprendizaje. Como consecuencia, el análisis científico no tiene tanto la vocación de resolver los problemas como de multiplicarlos. Ello está alimentado por la preferencia del sistema por la innovación, de modo que cualquier realidad pueda convertirse en objeto de análisis. La unidad formada por el binomio descomposición/recomposición se convierte en la condición de la aparición de un nuevo saber. La capacidad de resolución casi sin límites así desarrollada por el sistema científico 
hace visible unas posibilidades casi infinitas para la sociedad, sabiendo que solamente se realiza una parte de lo posible. ${ }^{5}$

2. La función del sistema mediático no es ni el incremento del saber, ni la socialización, ni la educación de los individuos. Produciendo información, los medios de comunicación crean un horizonte de incertidumbre autogestionado que debe ser compensado por información adicional. Por una parte, se caracterizan por una preferencia por la información, teniendo en cuenta que ésta pierde su carácter de sorpresa con su publicación, es decir que corre el riesgo permanente de convertirse en no-información, lo que exige su sustitución constante. La función de los medios de comunicación consiste entonces en la producción y en el tratamiento de la capacidad de irritación del sistema social, sabiendo que la irritación es el proceso a través del cual unos acontecimientos que se han producido en el entorno encuentran cierta resonancia en el sistema. Por otra parte, los medios de comunicación generan realidad, pero se trata de una realidad que no busca el consenso. Para Luhmann, la estabilidad de la sociedad se fundamenta, en primer lugar, en su capacidad de producir objetos que pueden ser utilizados para alimentar la comunicación. Intentar asegurar esta continuidad de la comunicación basándose en garantías, tales como el consenso o el contrato, sería demasiado arriesgado, puesto que estos objetos surgen de la comunicación. La existencia de semejantes objetos en las sociedades modernas es obra de los medios de comunicación, ya que la comunicación individualizada que propone puede comunicarse ella misma sin prejuicio y dejar en manos de una comunicación complementaria el quehacer de expresar su posición con respecto a la primera comunicación.

3. El sistema religioso administra la inevitabilidad de la contingencia y domestica el problema planteado por el hecho de que cualquier determinación, es decir cualquier selección o toma de decisión, produce una nueva indeterminación. «La religión se inspira de la simultaneidad de la determinación y de la indeterminación así como de la necesidad de la contingencia». ${ }^{6}$ Añade posteriormente la idea de que el sistema religioso intenta presentar en la comunicación la diferencia entre lo que es observable y lo que no lo es. Para Luhmann, cualquier comunicación hace referencia a algo observable, en la medida en que realiza una distinción entre lo que está determinado y lo que queda excluido. Esta diferencia observable/no observable es el problema central de la religión. Toda la

5 Luhmann, N. 1994: «La science de la société. Questions à Niklas Luhmann», Recherches sociologiques, $\mathrm{n}^{\circ} 43, \mathrm{p} .364$.

6 Luhmann, N. 1977: Funktion der Religion. Frankfurt am Main: Suhrkamp, p. 33. 
comunicación en su seno se encuentra estructurada por la cuestión de la unidad de esta diferencia.

4. La función del sistema político es la producción de decisiones colectivamente coactivas. Las decisiones suponen elecciones. Por lo tanto, la política define el futuro como la incógnita y lo indeterminado como lo que está enfrentado. ${ }^{7}$ La capacidad de producir decisiones colectivamente coactivas está vinculada a la ocupación de un cargo político. Porque los cargos electos deben lograr la aprobación del público para acceder a dichos cargos, deben satisfacer sus intereses, proponer mejoras y poner en evidencia los problemas. La dificultad se agudiza cuando se privilegia una perspectiva a largo plazo donde cada uno intenta asegurarse en el presente lo que podría necesitar en el futuro. A escala de la sociedad, la reducción de la rareza es precisamente lo que aumenta la rareza. No obstante, las necesidades siguen estando en el entorno del sistema y no pueden ser programadas.

5. El sistema económico solo puede programar sus propias operaciones, es decir los pagos que realiza a través de los precios. Los precios están determinados por lo que las personas están dispuestas a pagar en el mercado, lo que se encuentra determinado a su vez por la cantidad de dinero disponible. Dicho de otra forma, en la economía moderna plenamente diferenciada, no existe ninguna regulación exterior de los precios de tipo moral: el precio justo está fijado de manera auto-regulada por los procesos económicos, es decir por la dinámica de los mercados.

6. La función asumida por el sistema educativo es la transformación de los sistemas psíquicos singulares, de manera que éstos sean capaces de participar en cualquier forma de comunicación. Deviene necesario para la sociedad cuando la socialización no consigue asegurar el comportamiento adecuado. La peculiaridad del sistema educativo es, por lo tanto, que sus efectos se manifiestan, a diferencia de los demás subsistemas funcionales, en el exterior del sistema social.

7. La familia debe incluir la comunicación que tiene lugar en su seno entre todos los participantes. La persona no es para Luhmann el conjunto «sistema psíquico/sistema orgánico» que compone el ser humano, sino que es un punto de identificación en la comunicación y una unidad a la que se pueden atribuir ciertos actos. No obstante, contrariamente a los demás sistemas funcionales, en los que se participa de manera limitada, todo lo que se refiere a los participantes, todas sus acciones y experiencias, incluso las que tienen lugar fuera de la familia, son potencialmente

7 Luhmann, N. 2000: Die Politik der Gesellschaft. Frankfurt am Main: Suhrkamp, p. 104. 
pertinentes en la comunicación familiar. La comunicación se ve afectada por todo lo referente a los sistemas psíquicos de sus miembros. La función de la familia no consiste, por lo tanto, en socializar, fenómeno que se produce en cada interacción social en la medida en que los participantes aprenden por la observación recíproca o reaccionando a las expectativas de los demás. La familia es sobre todo el subsistema en el seno del cual el couplage estructural entre el sistema psíquico y el sistema social tiene lugar: la comunicación tematiza lo que comprenden y escuchan los sistemas psíquicos que participan en él.

Esta diferenciación presenta numerosas similitudes con la autonomización de las esferas de actividad descrita por Weber, puesto que los dominios se convierten cada vez más complejos y gozan de su propia lógica de acción. Tanto en Weber como en Luhmann, la ruptura con la moral holista de las sociedades tradicionales es la condición y la consecuencia de este proceso de diferenciación. En cada sistema en formación, «aparecen símbolos que tienen connotaciones a-sociales o por lo menos meta-morales, como, por ejemplo, la razón de Estado en el ámbito del poder, el beneficio en el dominio de la propiedad o la pasión enfermiza en el ámbito del amor» ${ }^{8}$ Ciertamente, el código moral, que se deshace en un momento dado de su fundamento en la religión, tiene cierta tendencia a invadir los subsistemas diferenciados multiplicándose como un parásito. Comparando la moral a una infección bacteriana, Luhmann concede que, «como las bacterias en el cuerpo, la moral puede también jugar un papel en los sistemas funcionales». ${ }^{9}$ A pesar de todo, no se puede sacar de la conformidad moral ni de la jerarquía de las categorías sociales, los medios para formar un sistema social.

No obstante, la teoría de Luhmann desplaza el modelo de la diferenciación presentado por Weber. La voluntad de ruptura se traduce en la semántica: si Differenzierung es el término utilizado por Weber para designar el proceso de diferenciación como división y descomposición, Luhmann recurre al término Ausdifferenzierung para poner el énfasis en la desvinculación de un subsistema con respecto a su entorno. Para Weber, la diferenciación es sinónimo de racionalización formal, subrayando la extensión de las reglas formales e impersonales del derecho moderno y la conmensurabilidad de la forma de dominación que prevalece en las sociedades modernas hacia las relaciones sociales, caracterizando así la diferenciación y, por lo tanto, la modernidad del pluralismo de los valores y la pérdida de sentido. Luhmann, sin embargo, rechaza cualquier referencia a los valores y al sujeto.

8 Luhmann, N. 1990: L'amour comme passion. Paris: Aubier, p. 47-48.

9 Luhmann, N. 1995: Gesellschaftsstruktur und Semantik. Frankfurt am Main: Suhrkamp, p. 431. 
Además, si para Weber, la racionalización equivale a la institucionalización de la racionalidad con respecto a un fin, la noción de función, para Luhmann, no tiene nada que ver con la finalidad de las acciones y de las instituciones. La función no sirve para la orientación del sistema como tal, ya que una operación no necesita conocer su función. Una función es solamente un enfoque elegido para realizar una comparación. Marca un problema de tal forma que múltiples soluciones pueden ser comparadas y el problema sigue vigente. Simultáneamente, la solución crea el problema que pretende resolver. En otros términos, la utilización de los conceptos de problema y de función se inscribe en una perspectiva que pretende problematizar de nuevo las instituciones, las selecciones y las soluciones establecidas, en beneficio de posibles alternativas y estableciendo hasta dónde es posible ir en la exploración de las variaciones sin hacer explotar el contexto funcional.

La diferenciación tampoco está pensada por Luhmann sobre el modelo de la división del trabajo entre esferas de actividad.

-Por una parte, la diferenciación no viene de arriba y no se produce bajo la forma de la división de un todo sobre la base de diferencias fundamentales. Tampoco se entiende como una compartimentación realizada y organizada por una entidad que continúa reservando un lugar a una totalidad armoniosa. Así, la descripción de la totalidad social no se reduce a la suma de las auto-descripciones de los subsistemas: cada uno reconstruye la sociedad a partir de su propia perspectiva sistema/ entorno. Es la razón por la cual, según Luhmann, los participantes en los diferentes subsistemas tienden a sobrevalorar sus posibilidades. Por ejemplo, los representantes del sistema político vinculan constantemente el código del poder con la moral y se presentan como los representantes del interés común.

-Por otra parte, Luhmann rompe con las representaciones funcionalistas de la diferenciación que la convierten en un conjunto de procesos acumulativos que se producen de manera casi automática. A su entender, la diferenciación se produce sobre la base de realizaciones muy específicas en términos de evaluación, en donde el invento de la concentración del poder en cargos políticos ha permitido la diferenciación del sistema político. Pero si semejantes innovaciones constituyen condiciones para la diferenciación de un sistema, su emergencia no conduce necesariamente a la aparición de un subsistema.

-Por último, la teoría de Luhmann se distingue de los enfoques tradicionales de la diferenciación que ponen el énfasis en la unicidad de cada ámbito diferenciado, en la medida en que la teoría de los sistemas parte de la comparabilidad de los subsistemas. Cuestiones tales como el límite del sistema, de la función, del medio y de las formas, del cierre 
operativo, de la autopoiesis o de la observación, pueden tratarse en relación a cualquier sistema.

El caso del arte permite dar cuenta de la amplitud del proceso de desvinculación del sistema con respecto a su entorno. Para Luhmann, el sistema artístico es comunicación, lo que no significa que se autonomiza con el desarrollo de una comunicación sobre el arte, sino que el arte es un equivalente funcional del lenguaje, incluso cuando emplea los textos como medio artístico. La observación de obras de arte solo funciona si el observador descodifica la estructura de las distinciones de la obra y deduce de todo ello que el objeto debe su existencia a la intención de transformar la información. ${ }^{10}$ La obra de arte transporta informaciones no solamente en sí misma sino en relación con las demás obras, para permitir la perpetuación de la comunicación. En resumen, el sistema artístico comunica gracias a las obras de arte. La comunicación es insensible y extranjera a la comunicación que circula en el mundo político o religioso.

La descripción Luhmanniana de la diferenciación de los subsistemas constituye una radicalización del modelo tradicional de la autonomía de las esferas de valores y de conocimiento. Esta radicalización deviene posible gracias a la noción de autopoiesis.

\section{AutOPOIESIS Y AUTO-REFERENCIA}

Las características del cierre recíproco que organizaban las relaciones entre el sistema social y el sistema psíquico así como entre el sistema social y el sistema vivo se encuentran duplicadas con el concepto de autopoiesis en el sistema social que rige las relaciones ente los subsistemas. Inicialmente, Luhmann ha utilizado la noción de auto-referencia para intentar aprehender esta propiedad de los sistemas que consiste en crearse ellos mismos, para proponer una definición de los sistemas como entidades constituidas por operaciones. Desvinculado de su soporte tradicional, el concepto de auto-referencia se refiere a la unidad que un elemento, un proceso o un sistema, es para sí mismo, es decir independientemente de los cortes que pueden ser realizados por la observación de los demás. El concepto contiene también la idea de que la unidad solo puede venir de una operación, que debe estar producida, que no preexiste y que está vinculada a un individuo, una sustancia o una idea de su propia operación. ${ }^{11}$ Para los sistemas sociales, esta idea se traduce por la neutralización de todos los antecedentes y condiciones de pertenencia a un dominio que no se fundamenta en este ámbito. Dicho de otra forma, la comunicación no está programada desde el exterior.

10 Luhmann, N. 1995: Die Kunst der Gesellschaft. Frankfurt am Main: Suhrkamp, p. 70 .

11 Luhmann, N. 1984: Soziale Systeme : Grundrib einer allgemeinen Theorie. Frankfurt am Main: Suhrkamp, p. 36. 
Retomando el ejemplo del arte, incluso unos objetos que pertenecen al ámbito doméstico, son dignos de convertirse en objetos artísticos. Es suficiente que formen parte de una comunicación en el seno del sistema artístico para beneficiarse de un estatus de objeto de arte y no necesitan referirse a la trascendencia. Asimismo, el derecho no consiste en adaptar lo que es justo por naturaleza porque preexiste a cualquier comunicación jurídica, aunque esté constituido por unas reglas jurídicas. En el amor, las cualidades esenciales y altamente valoradas, tales como la belleza o la virtud, hacia las cuales se orienta el sentimiento amoroso, según la semántica amorosa de los siglos XVII y XVIII, desaparecen, para banalizarse y obedecer a la biología. El amor se convierte entonces en su propia finalidad. ${ }^{12}$

El concepto de autopoiesis complejiza y radicaliza todavía más el proceso. Implica, en primer lugar, que las fronteras del sistema están producidas por sus propias operaciones. En otros términos, la identidad sistémica es una identidad en acto y solo está constituida por operaciones de distinción que dibujan su contorno. Por lo tanto, un sistema autopoiético es un proceso que se construye y disuelve continuamente, de modo que sea imposible descomponerlo en elementos simples. Además, el sistema se enfrenta en cada instante al problema de su perpetuación, a la cuestión de saber cómo seguir y cómo hacer para que una operación suceda a otra. La reproducción autopoiética del sistema no es la repetición idéntica de lo mismo sino la creación constante de nuevos elementos vinculados a los precedentes.

Es la razón por la cual un sistema necesita desarrollar sus estructuras. Una estructura permite al sistema asegurar y regular la reproducción de sus elementos. Una estructura consiste en la selección de posibilidades restringidas de puesta en relación de elementos ${ }^{13}$ de modo que la incertidumbre inducida por el futuro se encuentre reducida. Los temas constituyen un tipo mayor de estructura para un sistema formado únicamente por comunicación. Ordenan los procesos de comunicación introduciendo coacciones sobre la secuencia de comunicación en curso e imponiendo su propia selectividad. Para perpetuarse, la comunicación necesita que la selección sea constante, lo que garantiza especialmente la aparición y la prosecución de un tema. Los temas organizan la memoria de la comunicación. Reúnen las contribuciones en series de elementos que se pertenecen mutuamente, de manera que se pueda distinguir, a lo largo de la comunicación, si un tema ha sido retenido o cambiado. Estos temas pueden ser estructurados posteriormente, ya que la selección de los temas se

12 Luhmann, N. 1990: L'amour comme passion. Paris: Aubier, p. 175-176.

13 Luhmann, N. 1984: Soziale Systeme : Grundrib einer allgemeinen Theorie. Frankfurt am Main: Suhrkamp, p. 384. 
convierte en la función propia de las estructuras más complejas en el seno de los diferentes subsistemas.

El uso del concepto de autopoiesis induce además que un sistema es incapaz de alcanzar su entorno gracias a estas operaciones. Sin embargo, esta característica no conlleva un cierre absoluto ante el medioambiente, pero la regulación y la selección, por el mismo sistema, establece lo que es pertinente para él en su entorno y ante lo cual reacciona. El fenómeno recuerda los trabajos del biólogo Jakob von Uexküll sobre la relación de los animales con su entorno. Según él, se limitan a lo que es pertinente para ellos. Luhmann aplica al sistema esta propiedad que consiste en percibir y utilizar en su entorno lo que es pertinente para él. No se interpreta como una pobreza sino como un control por parte del sistema de la complejidad. «Los sistemas autopoiéticos son soberanos hacia la creación de identidades y de diferencias. Evidentemente, no crean por sí mismos un mundo material. Presuponen otros niveles de realidad. Pero, sean cuales sean las identidades y las diferencias que utilizan, siempre son aquellas que han trazado ellos mismos».

Por lo tanto, el entorno del sistema solo encuentra su unidad a través del sistema y únicamente encuentra su opacidad por el sistema. Si el entorno reúne todo lo que el sistema puede disponer vía la reproducción autopoiética, la relación a los demás sistemas está concernida por esta imposibilidad estructurada.

\section{ConClusión}

Recordemos que este artículo se ha interesado por la obra de Niklas Luhmann, obra profundamente original y difícilmente clasificable. Este sociólogo alemán ha elaborado una teoría ambiciosa y coherente que describe la sociedad moderna como un sistema. Constituido, no tanto por individuos, sino por comunicaciones, se diferencia en subsistemas funcionales cerrados a través de códigos especializados: los sistemas político, económico, religioso, artístico o jurídico. Inspirándose de autores, teorías y disciplinas muy diferentes (biología del conocimiento, cibernética, lógica, lingüística, teoría de la comunicación, fenomenología, filosofía de la deconstrucción) Niklas Luhamman ha construido una de las obras más fecundas y singulares del siglo XX. El objetivo de este artículo ha sido precisamente estudiar su teoría de los sistemas.

En la continuidad de su teoría de los sistemas, Luhmann se ha interesado por la sociedad mundial. Efectivamente, por la simple elucidación de las lógicas impuestas por su teoría de la sociedad y de la comunicación, ha construido una concepción audaz de la globalización. Lejos de constituir un horizonte o una consecuencia inevitable de una mutación reciente, la sociedad mundial emerge al inicio de la modernidad con el paso a una sociedad diferenciada funcionalmente. La aparición de los diferentes subsistemas, tales como la economía, la ciencia, la religión, el arte o la intimidad, implica «la existencia de diferentes 
fronteras, no solamente para cada uno de ellos sino también para la sociedad en su conjunto». A partir de este instante, la economía es mundial y tanto la ciencia como la sociedad devienen co-extensivas a la comunicación. Por lo tanto, la existencia de una ciencia mundial depende de los medios de comunicación que realizan su integración comunicativa. Sin ellos no podría existir; no podría construir las conexiones necesarias entre las comunicaciones sobre la base de una producción puramente manual del sentido.

Luhmann repite que no es realista pensar, por ejemplo, que la sociedad brasileña sea diferente de la tailandesa, lo que no significa que no tome en consideración las especificidades regionales. Pero, una teoría sociológica que pretende explicar estas diferencias no debería aceptarlas como datos y variables independientes sino que debería intentar comprender por qué aparecen. En este sentido, el concepto de sociedad mundial es considerado fuera de cualquier referencia a la idea de integración o de lazo social. No presupone ninguna forma de identidad común y no es considerada como obligatoria para sus miembros. En este sentido, no existe homogeneidad alguna en los estilos y modos de vida. Son las diferencias y no las identidades las que ofrecen la posibilidad de percibir y tratar la información, y, por lo tanto, permiten la prosecución de la comunicación.

Para Luhmann, la globalización no es una realización de la política porque el único sistema que no es mundial es precisamente el político, que se caracteriza por el hecho de estar sometido a la diferenciación regional. No obstante, se desarrolla cada vez más una política mundial, que está orientada hacia unos intereses no regionales y que solo se sirve de los Estados para recoger las exigencias de la sociedad mundial en materia de adaptaciones y de reestructuraciones regionales. Esta política constituye una auto-descripción y de una comunicación delimitada territorialmente..$^{14}$ Luhmann indica que una limitación regional es la mejor forma para optimizar el funcionamiento del sistema político.

Su concepción de la sociedad mundial traduce una preocupación normativa. Ilustra el vínculo que mantiene el sociólogo alemán con la teoría, porque el hecho de hablar de la sociedad mundial le permite concebir las estructuras de los países en vía de desarrollo como una consecuencia de su racionalidad, que tiende a fortalecer las distinciones. Asimismo, concibe la sociedad mundial como una perspectiva teórica que permite pensar una condición global que enfrenta distintas identidades. Hace posible el cuestionamiento sobre la siguiente pregunta: jla condición general de la negligencia global no estimula la búsqueda de identidades regionales o étnicas? El argumento de la desigualdad es un argumento a favor de la sociedad mundial, ya que los diferentes niveles de

14 Hayoz, N. 1997: L'étreinte soviétique: aspects sociologiques de l'effondrement programmé de l'URSS. Genève: Droz, p. 4. 
desarrollo exigen una explicación. Lo que supone partir de la unidad del sistema social que produce estas diferencias. Por lo tanto, una teoría debe partir de la hipótesis de una sociedad mundial y explorar ¿cómo y porqué esta sociedad tiene cierta tendencia a mantener e incluso a aumentar las desigualdades y las diferencias regionales?

Eguzki Urteaga es Profesor de Sociología en la Universidad del País Vasco e Investigador en el Centro de investigación IKER, laboratorio asociado al CNRS francés. Es Doctor en Sociología por la Universidad Victor Segalen Buerdos 2 y Licenciado en Historia mención Geografía por la Universidad de Pau y de los Países del Adur, es autor de 20 libros entre los cuales se encuentran: Sociología moderna y contemporánea (2002), La politique linguistique en Pays Basque (2004), La nouvelle gouvernance en Pays Basque (2004), La question basque en France (2004), La sociología de la complejidad (2005), La coopération transfrontalière en Pays Basque (2007), La politique d'immigration du gouvernement basque (2008) y Les Plans Locaux d'Immigration en Espagne (2008) así como varios artículos universitarios tanto en Europa como en Canadá. Es igualmente Director del Grupo de Investigación de Estudios Vascos, Director de Colección en la editorial de París Mare et Martin y Vice-Presidente de la Sociedad de Estudios Vascos - Eusko Ikaskuntza.

Dirección postal:

Universidad del País Vasco, Departamento de Sociología 1, Los Apraiz, 2, 01006 Vitoria

Correo electrónico: eguzki.urteaga@ehu.es 
